\title{
Interactive comment on "Rainfall intensity bursts and the erosion of soils: an analysis highlighting the need for high temporal resolution rainfall data for research under current and future climates" by David L. Dunkerley
}

\section{Anonymous Referee \#1}

Received and published: 6 February 2019

General comments: This discussion paper deals with the issue of rainfall intensity, and how values may be misleading when aggregated on daily or even hourly timescales. Rainfall intensity realistically changes at much shorter timescales on the order of seconds, and high intensity bursts embedded within longer periods of less intense rainfall can be very important for soil erosion. The paper first discusses typical measurement and averaging intervals for rainfall data, and the potential disconnect between these averaged values and features of water erosion of soils and other earth surface processes. The author then introduces an example based on tipping bucket field data from 2 sites, 
one arid and the other very wet. The datasets span several years of 1-second resolution data, and the focus here is on inter-tip times (ITT) which provide a reference of rainfall intensity at the shortest possible time scale. This example detects some very high intensity bursts at both sites, with several features that vary between sites. Given an hourly or daily resolution, this data would show very different rainfall intensities from those observed on short timescales. The paper then finishes with a discussion of how rainfall influences soil erosion through ponding and other mechanisms, the potential influence of climate change, and other ways in which rainfall intensity is important to understand at fine timescales.

Overall, I found this paper to be interesting and important, as it provides an illustrative example of rainfall intensity at different timescales and promotes a better understanding of this feature of rainfall. I have several comments listed below on how this paper could be improved in terms of readability and the presentation of the rainfall analysis. In the discussion parts of the narrative, the author references many previous studies on rainfall intensity to explain various aspects of averaging and its importance. This causes the narrative to often jump from topic to topic, each with a lot of details, and I feel there could be smoother connections in many discussion paragraphs at the beginning and end of the paper. I would recommend going through these paragraphs and making sure there is a main point, that each study cited then promotes. Otherwise, the paper is nicely written. I also have several minor comments on the presentation of the data analysis, mainly related to the representation of the results in the table, which are detailed below.

Specific comments: There are a lot of tables presented, and I think the results would be more interpretable in Figures instead. For example, the results of Table 1 could instead be integrated into Figure 1. In Figure 1, the panels should have the same yaxis ranges to better show differences in intensity, and similarly the $x$-axis ranges could be made more clear by using hours instead of Julian day (or in addition to Julian day). I realize the analysis was done using JD, but it would be much easier to read the plot if

ESurfD

Interactive comment
Printer-friendly version

Discussion paper 
a more typical timescale was used for plotting. The duration of each event, the depths of the events, the mean intensity, and the peak intensity then become more apparent in Figure 1 - with a dotted horizontal line for the mean, a cumulative line for the total depth, etc. Finally, Figure 1 is introduced before the sites, which makes the results a bit confusing - it would be good to briefly mention the different sites in the text just before introducing the figure, and then going into details later.

ESurfD

Interactive comment

The paragraph starting on Line 6 of page 6 is very technical, and I think some of this information could be supplementary or unnecessary, as it breaks the flow into the data analysis at the field sites. The MIT definition is important, and the paper could use an additional sentence explaining this at the end.

Page 6 line 20: does $\mathrm{N}$ refer to total number of events during the study period? Page 7 line 17: I would add "due to differences in the tipping bucket sizes," at the beginning of the sentence here, as to why the values are different between FG and MM Page 7 line 20: the term "nominated value" is strange - maybe say "a chosen threshold value"? Table 2 and Table 3: a separation (e.g. bold line) would be useful between the third and fourth column. Alternately, as in Figure 1, I think this could be better shown in a figure. For example, each exceedance duration is embedded into the next longer duration, if I am interpreting it correctly, so a sort of cumulative plot could show all the same information more succinctly. The first 3 columns here seem redundant, and could be given with an equation related the ITT, the min intensity, and the tips at that intensity instead of the table.

During the middle part of the paper which focuses on the results, the reader could use some reminders of the acronyms at intervals (e.g. on page 9, line 15, remind us of the site names)

Printer-friendly version

Figure 2: This result could be made more powerful by combining the lines onto a single plot, so the magnitudes could be compared. A logarithmic scale could be used on the $y$-axis to distinguish the magnitudes, and show that the 1 hour aggregation loses 
an order of magnitude in intensity compared to the unaggregated values. Table 4-5: similar comment for this in Figure form instead (a pie chart, bar chart, etc since it is segmenting the ITTs into their time ranges), and a side-by-side comparison between the two sites would be more useful to talk about this feature.

Figure 3: squares and diamonds are mentioned in the caption - but I see circles and triangles in the Figure (a legend in the Figure would be useful) For this Figure and Figure 4, I think Equations 1-5 could be removed and placed inside the appropriate subfigure panels.

Table 7 is referenced in the text but I don't see it in the manuscript?

Page 17 line 14: the phrase "duration have durations of hours" is very confusing, I had to read it a few times before it was clear. Page 17 line 25: "relationship" Page 17 line 22: "hourly"? Page 17 line 29: The "I_30" index is not well-defined here, and this type of index is referred to many times in the discussion part of the paper - would benefit from a definition here.

Page 19: This paragraph is an example of one with a lot of information contained in it. It could use improvement of language to synthesize and highlight the main issue of erosion on post-fire landscapes.

Page 20, line 21: incomplete sentence Page 21 line 29: "present field site" - be more specific here and indicate specific sites In the conclusion section, when you say "the brief snapshot of soil erosion mechanisms" - I feel that the previous sections are actually more broad in terms of not only soil erosion, but climate change, urban flooding, and other aspects of rainfall and climate. I think this indicates that the previous sections could be better tied in with the main topic of rainfall intensity and soil erosion, e.g. in the section on climate change and the $\mathrm{CC}$ relationship and how rainfall intensity may be influenced, bring the discussion back to soil erosion. Otherwise, this could be done in the conclusion with a few extra sentences. Additionally in the conclusions section, it would be useful to refer back to the field data analysis in the context of this broader

ESurfD

Interactive comment
Printer-friendly version

Discussion paper 
discussion of how finer resolution rainfall data is needed to link between rainfall and soil erosion.

ESurfD

Interactive comment on Earth Surf. Dynam. Discuss., https://doi.org/10.5194/esurf-2018-94, 2019.

Interactive

comment 